\title{
Marcadores de Resiliência Infantil: Evidências de Validade para Estrutura Interna e Precisão
}

\author{
Karina da Silva Oliveira ${ }^{1}$ \\ ${ }^{1}$ Universidade Federal de Minas Gerais, Belo Horizonte, \\ MG, Brasil. \\ Tatiana de Cássia Nakano² \\ ${ }^{2}$ Pontifícia Universidade Católica de Campinas, Campinas, \\ SP, Brasil.
}

\author{
Evandro Morais Peixoto ${ }^{3}$ \\ ${ }^{3}$ Universidade São Francisco, Campinas, \\ SP, Brasil.
}

\begin{abstract}
Resumo: A resiliência tem sido considerada um construto importante para o enfrentamento de situações adversas. Em que pese sua relevância, nenhum instrumento brasileiro encontrase disponível para sua avaliação, especialmente na infância. Diante dessa lacuna, o objetivo deste estudo foi buscar evidências de validade baseadas na estrutura interna e na precisão do instrumento Marcadores de Resiliência Infantil (MRI), que busca verificar a presença ou ausência de indicadores de potenciais resilientes em crianças com idades entre oito e 12 anos. Participaram 461 crianças, das quais 223 eram meninas, de escolas públicas e particulares. Através do modelo Bi-factor, os resultados confirmaram o modelo teórico que serviu de base para sua construção, indicando a presença de seis fatores específicos e um fator geral (vulnerabilidade, coping, inteligência emocional, habilidade, bem-estar subjetivo, locus de controle) representados em 22 itens. Os coeficientes de alfa de Cronbach apontaram para adequação dos fatores Locus de controle, Copinge Total.É possível concluir que foram alcançadas evidências iniciais relacionadas à validade e à precisão do instrumento. Sugere-se que outros estudos sejam realizados a fim de compreender, de forma aprofundada, as qualidades psicométricas desse instrumento.
\end{abstract}

Palavras-chave: Resiliência, Construção de Instrumentos, Avaliação Psicológica.

\section{Children's Resilience Markers: Validity Evidence for Internal Structure and Reliability}

\begin{abstract}
Resilience has been considered a relevant psychological phenomenon for coping with adverse events. Despite its relevance, the Brazilian scenario still lacks an instrument for the evaluation of such phenomenon, especially regarding childhood. Aiming to bridge this scientific gap, this study searched for validity evidences based on the internal structure and reliability of the Child Resilience Markers (CRM), an instrument that seeks to verify the presence or absence of potential indicators of resilience in children aged between eight and 12 years old. The study was conducted with 461 children from public and private schools, 223 of whom were girls. Using the Bi-Factor model, the results confirmed the theoretical model that served as the basis for the construction of the instrument, indicating six specific factors (vulnerability, coping, emotional intelligence, ability, subjective well-being, locus of control) and a general factor represented into 22 items. Cronbach's alpha coefficients indicated the adequacy of the Locus of control, Coping, and Total factors. These findings evince the validity and reliability of the instrument, indicating the need for further studies to provide a deeper understanding of this instrument psychometric qualities.
\end{abstract}

Keywords: Resilience, Test Development, Psychological Assessment. 


\title{
Marcadores de Resiliencia Infantil: Evidencias de Validez para la Estructura Interna y Exactitud
}

\begin{abstract}
Resumen: La resiliencia se ha considerado un constructo importante para el enfrentamiento de situaciones adversas. A pesar de su relevancia, ningún instrumento brasileño se encuentra disponible para evaluarla, especialmente en la infancia. Ante esta brecha, el objetivo de este estudio fue buscar evidencias de validez basadas en la estructura interna y la exactitud del instrumento Marcadores de Resiliencia Infantil (MRI), que pretende verificar la presencia o ausencia de indicadores de potencial de la resiliencia en niños de edades comprendidas entre 8 y 12 años. Participaron 461 niños, de los cuales 223 son niñas, de escuelas públicas y privadas. Por medio del modelo Bi-Factor, los resultados confirmaron el modelo teórico que sirvió de base para su construcción, indicando la presencia de seis factores específicos y un factor general (vulnerabilidad, afrontamiento, inteligencia emocional, capacidad, bienestar subjetivo, locus de control) representados en 22 ítems. Los coeficientes de alfa de Cronbach apuntaron a la adecuación de los factores Locus de control, Coping y Total. Es posible concluir que se han alcanzado evidencias iniciales relacionadas con la validez y exactitud del instrumento. Se sugiere realizar más estudios para conocer, en profundidad, las cualidades psicométricas de este instrumento.
\end{abstract}

Palabras clave: Resiliencia, Desarrollo de Pruebas, Evaluación Psicológica.

Ao considerarmos as exigências presentes na sociedade moderna, é possível observar que os indivíduos são submetidos a situações que podem ser consideradas demandas complexas que envolvem o manejo de emoções negativas, frustrações e estresse (Faiad, Pasquali, \& Primi, 2016). Dentre os construtos envolvidos no processo de enfrentamento das adversidades, a resiliência se destaca como aquele que pode favorecer um desfecho positivo diante da situação problema (Masten, 2001, 2018).

Embora a literatura científica da temática seja marcada por inconsistências na forma como a resiliência é definida e medida (Masten \& Cicchetti, 2016), percebe-se que os autores defendem que este construto seja um fenômeno psicológico (Yunes, 2011) presente indistintamente nos indivíduos (Masten, 2001, 2014, 2018), podendo desenvolver-se e manifestar-se ao longo do ciclo vital (Fontes, 2010; Infante, 2007; Masten, 2001; Poletto, Wagner, \& Koller, 2004), sempre que o indivíduo for submetido a uma condição adversa (Luthar, Cicchetti, \& Becker, 2000), real ou percebida (Castillo, Castillo-López, López-Sánchez, \& Dias 2016). O enfrentamento dessa condição envolverá a utilização de recursos individuais e sociais (Castillo et al., 2016; Masten, 2001, 2014; PrinceEmbury, 2006, 2010; Yunes, 2011), a fim de apresentar um desfecho positivo (Infante, 2007; Masten, 2001; Reppold, Mayer, Almeida, \& Hutz, 2012).

Quando tomamos as características dos diferentes estágios do ciclo vital, é possível observar que a infância é uma etapa importante para a aquisição de habilidades físicas, cognitivas e emocionais (Oliveira et al., 2012), marcada por um percurso de desenvolvimento que não se mostra linear e nem sempre tranquilo, mas cunhado por situações potencialmente adversas (Masten, 2014). Consequentemente, o estudo dos riscos de curto e longo prazo à saúde e ao bem-estar de crianças que vivenciaram experiências adversas, especialmente aquelas prolongadas, cumulativas e que ocorrem em períodos importantes do desenvolvimento, tem reforçado um interesse global na investigação da resiliência na infância (Masten \& Barnes, 2018).

Na criança, a resiliência dependerá dos recursos e suporte disponíveis, especialmente provindos dos sistemas com os quais a criança interage (Masten, 2018). Nesse sentido, os resultados desses eventos adversos poderão ser influenciados por fatores como a gravidade do evento, o contexto vivenciado antes e depois dele, o período desenvolvimental, as diferenças individuais e os recursos familiares, escolares e sociais, assim como o suporte externo que a família recebe (Masten, 2021). Nessa fase, os estudos com 
foco na resiliência que visam identificar quais fatores apresentam suporte empírico mais relevante para a promoção da resiliência mostram-se importantes, visto que o conhecimento derivado pode ser usado como base para o desenvolvimento de trabalhos voltados à prevenção e à elaboração de políticas públicas (Yule, Houston, \& Grych, 2019).

Desse modo, a avaliação da resiliência nesse período pode auxiliar no processo de identificação de fatores de risco e proteção, assim como no reconhecimento de habilidades e repertórios que podem ser desenvolvidos por meio de estratégias de intervenção. Visando contribuir com esse processo, um instrumento denominado Marcadores de Resiliência Infantil (MRI) (Oliveira, \& Nakano, 2020) teve seu processo de desenvolvimento iniciado com base na constatação de que, indiferente à relevância do construto da resiliência para o enfrentamento de situações adversas, (Oliveira \& Nakano, 2018; Reppold et al., 2012) não existem, em âmbito nacional e até o momento, instrumentos para a mensuração da resiliência que estejam disponíveis para uso profissional e que estejam aprovados pelo Sistema de Avaliação de Testes Psicológicos (SATEPSI).

Apesar da existência de diferentes modelos teóricos para compreensão desse construto, o modelo de Castillo et al. (2016), que entende o construto por meio de recursos pessoais envolvidos na expressão da resposta resiliente, foi adotado como base teórica para o instrumento aqui apresentado. Para esses autores, a resiliência deve ser entendida como o resultado da relação intrínseca entre seis elementos fundamentais, que proporcionariam o fortalecimento de forças e virtudes (Masten, 2014, 2018; Prince-Embury et al., 2017). A seleção desse modelo foi embasada na necessidade de um modelo teórico que apresentasse potencial para uma compreensão mais objetiva do construto, de maneira a possibilitar o desenvolvimento de uma medida que auxilie o processo de identificação de potenciais resilientes, assim como o fortalecimento de características individuais.

Os seis elementos fundamentais desse modelo de resiliência são: a) vulnerabilidade, capacidade de antecipar, identificar, resistir e se recuperar de uma ameaça; b) coping, que se relaciona à expressão comportamental da resiliência, envolvendo o enfrentamento positivo diante da situação adversa; c) inteligência emocional, que seria a capacidade de reconhecimento, compreensão e regulação das emoções; d) bem-estar subjetivo, que diz respeito à avaliação que se faz de sua própria história e consequente satisfação; e) locus de controle, capacidade de manejar seus próprios comportamentos a fim de alcançar resultados finais almejados, em especial os acadêmicos; e f) habilidade, ou seja, a busca por excelência na execução de tarefas (Castillo et al., 2016).

Considerando o público-alvo, crianças e pré-adolescentes, algumas escolhas relacionadas ao formato do instrumento foram tomadas. A primeira delas envolveu a decisão de que o MRI não se caracterizaria por ser uma escala, uma vez que neste tipo de tarefa solicita-se que o avaliando avalie seu nível de habilidade, selecionando um valor que represente a intensidade com que o conteúdo daquela afirmativa se encontra presente em seu comportamento, de modo que tal tarefa pode se mostrar bastante complexa para ser realizada por crianças (Reppold et al., 2012). Por isso, o instrumento foi construído em formato de um teste de julgamento situacional, avaliando o potencial resiliente por meio de histórias que expressam diferentes níveis de habilidades e diferentes possibilidades de enfrentamento de uma situação adversa. Desse modo, o nível de competência/habilidade resiliente selecionado pelo respondente, considerando o caráter desenvolvimental deste construto, assim como a faixa etária almejada, poderá ser hipotetizado como uma expressão de seu potencial resiliente. Maiores detalhamentos serão apresentados por ocasião da apresentação do instrumento na seção referente à metodologia.

Considerando que durante o processo de desenvolvimento de uma medida psicológica é importante que sejam conduzidos diferentes estudos a fim de verificar as qualidades psicométricas do instrumento que está sendo construído, Ambiel e Carvalho (2017) destacam que as primeiras evidências a serem alcançadas devem ser as de validade baseadas na estrutura interna. Isso porque, ainda segundo os autores, este tipo de investigação visa verificar a adequação dos itens do instrumento em relação ao modelo teórico adotado. Para isso, o pesquisador deve conduzir estudos que possibilitem a compreensão dos diferentes agrupamentos dos itens, de modo que, a partir dessa observação empírica dos dados, será possível inferir a existência de um traço latente ou ainda do construto investigado (Primi, 2011).

Ainda que existam diferentes formas de investigação desse tipo de evidência de validade, no estudo aqui apresentado foi selecionada a Análise Fatorial Confirmatória (AFC). A opção por esse método se deu em função do fato de o instrumento ter sido construído com base em um modelo teórico de resiliência já conhecido na literatura científica sobre o construto, 
que entende que a resiliência pode ser observada por meio da relação intrínseca entre seis elementos fundamentais (Castillo et al., 2016). Nesse sentido, a AFC permite que o pesquisador realize o teste confirmatório da estrutura psicométrica do instrumento desenvolvido (Pilati \& Laros, 2007), bem como verifique a legitimidade da representação teórica do construto, que será operacionalizado por meio do instrumento investigado (Pasquali, 2011).

Outro aspecto importante a ser investigado ao longo do processo de construção de uma medida psicológica, refere-se à compreensão da precisão do instrumento. Segundo Namdeo e Rout (2016) e Primi (2011), estudos dessa natureza são valiosos ao longo do processo de desenvolvimento de medidas psicológicas, pois relacionam-se à capacidade de identificar o quanto um conjunto de itens é capaz de diferenciar examinandos com variados graus de habilidade mensurada pelo instrumento (Zanon \& Hauck Filho, 2015). Pasquali (2011) aponta para a existência de diferentes técnicas de avaliação da precisão de um instrumento, de forma que foi selecionada, para este estudo, a análise dos coeficientes de Alfa de Cronbach, uma medida de precisão objetiva amplamente utilizada que auxilia na compreensão da homogeneidade de um determinado grupo de itens (Ambiel \& Carvalho, 2017; Tavakol \& Dennick, 2011). Portanto, diante da relevância do construto da resiliência, seu potencial para contribuir com processos de avaliação psicológica, assim como considerando as lacunas presentes no cenário nacional, especialmente quanto a sua avaliação junto à infância, o presente estudo buscou investigar evidências iniciais de validade baseadas na estrutura interna e precisão do instrumento MRI.

\section{Método}

\section{Participantes}

Foram considerados participantes deste estudo crianças cujas idades estavam compreendidas no intervalo de oito a 12 anos, que estivessem devidamente matriculadas em escolas regulares de Ensino Fundamental I e II, cujos pais previamente autorizassem suas participações, por meio de Termo de Consentimento Livre e Esclarecido e que assentissem voluntariamente suas colaborações para a pesquisa. Diante desses critérios, contou-se com a participação de 461 crianças, das quais 223 (48,37\%) eram do sexo feminino, com idades entre oito e 12 anos ( $M=9,75$; $\mathrm{DP}=1,25)$. Quanto ao tipo de escola dos participantes, 413 eram alunos de escolas públicas e 48 eram provenientes de escola particular, localizadas em cidades da Região Metropolitana de Campinas e da cidade de São Paulo. O detalhamento das características sociodemográficas da amostra é apresentado na Tabela 1. Importa destacar que a amostra foi composta por conveniência. Ao longo do processo de aplicação, não ocorreram recusas ou desistências.

Tabela 1

Detalhamento sociodemográfico dos participantes.

\begin{tabular}{lcccccc}
\hline & \multicolumn{2}{c}{$\begin{array}{c}\text { Sexo Feminino } \\
(\mathrm{n}=223)\end{array}$} & \multicolumn{2}{c}{$\begin{array}{c}\text { Sexo Masculino } \\
(\mathrm{n}=238)\end{array}$} & \multicolumn{2}{c}{$\begin{array}{c}\text { Total } \\
(\mathrm{n}=461)\end{array}$} \\
\cline { 2 - 7 } & $\mathrm{F}$ & $\%$ & $\mathrm{~F}$ & $\%$ & $\mathrm{~F}$ & $\%$ \\
\hline Tipo de Escola & & & & & & \\
$\quad$ Pública & 198 & 88,79 & 215 & 90,34 & 413 & 89,59 \\
Particular & 25 & 11,21 & 23 & 9,66 & 48 & 10,41 \\
Total & 223 & 100,00 & 238 & 100,00 & 461 & 100,00 \\
Ano Escolar & & & & & & \\
$2^{\circ}$ ano & 4 & 1,79 & 10 & 4,20 & 14 & 3,04 \\
$3^{\circ}$ ano & 65 & 29,15 & 58 & 24,37 & 123 & 26,68 \\
$4^{\mathbf{0}}$ ano & 58 & 26,01 & 45 & 18,91 & 103 & 22,34 \\
$5^{\mathbf{0}}$ ano & 53 & 23,77 & 64 & 26,89 & 117 & 25,38 \\
$6^{\circ}$ ano & 42 & 18,83 & 59 & 24,79 & 101 & 21,91 \\
$7^{\mathbf{0}}$ ano & 1 & 0,45 & 2 & 0,84 & 3 & 0,65 \\
Total & 223 & 100,00 & 238 & 100,00 & 461 & 100,00 \\
\hline & & & & & continua...
\end{tabular}




\begin{tabular}{ccccccc}
....continuação & \multicolumn{2}{c}{$\begin{array}{c}\text { Sexo Feminino } \\
(\mathrm{n}=223)\end{array}$} & $\begin{array}{c}\text { Sexo Masculino } \\
(\mathrm{n}=238)\end{array}$ & \multicolumn{2}{c}{$\begin{array}{c}\text { Total } \\
(\mathrm{n}=461)\end{array}$} \\
\cline { 2 - 7 } & $\mathrm{F}$ & $\%$ & $\mathrm{~F}$ & $\%$ & $\mathrm{~F}$ & $\%$ \\
\hline Idade & & & & & & \\
8 & 47 & 21,08 & 42 & 17,65 & 89 & 19,31 \\
9 & 66 & 29,60 & 53 & 22,27 & 119 & 25,81 \\
10 & 48 & 21,52 & 65 & 27,31 & 113 & 24,51 \\
11 & 42 & 18,83 & 52 & 21,85 & 94 & 20,39 \\
12 & 20 & 8,97 & 26 & 10,92 & 46 & 9,98 \\
Total & 223 & 100,00 & 238 & 100,00 & 461 & 100,00 \\
\hline
\end{tabular}

\section{Instrumento}

O instrumento Marcadores de Resiliência Infantil (MRI) (Oliveira \& Nakano, 2020) é uma medida psicológica que objetiva avaliar o potencial resiliente em crianças com idades entre 8 e 12 anos. Foi desenvolvido a partir do modelo teórico proposto por Castillo et al. (2016), no qual os autores defendem que a resiliência é o produto da interação intricada entre seis elementos fundamentais: vulnerabilidade, coping, inteligência emocional, bem-estar subjetivo, locus de controle e habilidade. O material caracteriza-se por ser um teste de julgamento situacional, composto por 27 itens que são apresentados em forma de breves histórias ilustradas.

Em cada história, há uma personagem principal, ora Nino, e ora Nina. A idade de tais personagens corresponde à idade do respondente. Em cada item, a personagem principal encontra-se em uma situação desafiadora representativa do cotidiano infantil. A história é interrompida no momento em que a personagem deve apresentar um desfecho para a situação problema, e cabe ao avaliando selecionar, entre três opções de desfecho, aquela que mais se aproxima ao que ele faria caso fosse a personagem principal. A pontuação das alternativas varia entre 0 e 2 , de acordo com o desfecho selecionado $(0$ ponto $=$ resposta não adequada; 1 ponto $=$ resposta neutra ou que não resolve o problema integralmente; 2 pontos = resposta adequada $\mathrm{e}$ resiliente). Tais opções são apresentadas de forma aleatória nos itens do instrumento, para que um padrão de respostas não seja estabelecido. Tal medida busca evitar, dentro do possível, minimizar a possibilidade que o respondente perceba um padrão, bem como um possível viés de resposta (como a tendência a marcar o item do meio ou dos extremos).
Até o momento, uma série de estudos já foi conduzida com o instrumental (Oliveira \& Nakano, 2020), dentre as quais a validade de conteúdo foi investigada por meio da consulta a 16 especialistas, que avaliaram a adequação teórica do item de acordo com o modelo teórico adotado. Esses especialistas também foram responsáveis por estabelecer o sistema de pontuação para as alternativas de cada história.

Após essas ações, a adequação do MRI à população-alvo foi investigada por meio de um estudo piloto dividido em dois momentos. No primeiro momento, dois grupos de cinco crianças, um com idade entre oito e nove anos e os demais com 11 e 12 anos, foram convidados a avaliar os itens e a folha de respostas do MRI. No segundo momento, dois grupos com as mesmas idades também foram convidados a avaliar a adequação do material do MRI. Essas ações mostraram algumas modificações que poderiam melhorar a aplicabilidade e a compreensão do instrumento, de maneira que todas as sugestões foram acatadas na versão aqui apresentada. Os resultados também apontaram para a adequação do instrumento à faixa etária almejada. Por fim, as evidências de validade com base em critério externo foram investigadas, indicando que o instrumento foi capaz de diferenciar, dentre cinco grupos, aqueles compostos por participantes que apresentavam maior potencial resiliente (Oliveira, Nakano, \& Silva, 2019).

\section{Procedimentos}

Inicialmente o presente estudo foi submetido à avaliação do Comitê de Ética em Pesquisa com Seres Humanos, tendo sua execução aprovada (CAAE 66606517.5.0000.5481). Três instituições pertencentes à Região Metropolitana de Campinas e uma instituição 
da cidade de São Paulo autorizaram a condução da pesquisa. Cerca de 15 dias antes da coleta de dados, os pais/ responsáveis receberam o Termo de Consentimento Livre e Esclarecido e somente aqueles que devolveram o documento assinado puderam participar, sendo essencial também que o aluno assinasse o termo de assentimento. As aplicações foram realizadas pela primeira autora e ocorreram de forma coletiva em sala de aula. Em alguns casos, a aplicação ocorreu em outro ambiente adequado disponibilizado pela escola, como biblioteca e sala de apoio. Independentemente da condição, isto é, se aplicação em sala de aula ou outro espaço, as aplicações acontecem, para cada turma, em uma única sessão, com duração estimada de 50 minutos.

\section{Análise de dados}

Inicialmente foi construído um banco de dados contendo as informações sociodemográficas (sexo, idade, nível de escolaridade e tipo de escola), bem como as respostas a cada um dos itens de todos os participantes. Com auxílio do pacote estatístico Mplus 7.11, empregou-se a Análise Fatorial Confirmatória (AFC), com a finalidade de testar e comparar, por meio do teste de diferença do $\chi^{2}$, o modelo unifatorial, o modelo oblíquo, o modelo de segunda ordem e o modelo Bi-factor. Em seguida, após tomadas as decisões acerca do modelo fatorial, foram conduzidas as análises relacionadas à correlação entre os fatores do instrumento MRI e a análise dos coeficientes de alfa de Cronbach tanto para os fatores do instrumento, como para o total de itens. Para essas análises, utilizou-se o software Statistical Package for the Social Sciences (SPSS) versão 22.

Conforme orientações apresentadas na literatura especializada (Lara \& Alexis, 2014; Muthén \& Muthén, 2012) para a seleção do modelo fatorial, foram avaliados os seguintes índices: qui-quadrado $\left(\chi^{2}\right)$, graus de liberdade (gl), Comparative Fix Index (CFI), Tucker Lewis Index (TLI) e Root Mean Square Error of Approximation (RMSEA). Na interpretação dos dados, as recomendações de Pilati e Laros (2007) foram consideradas: valores relacionados ao CFI e ao TLI estejam compreendidos entre 0,90 e 0,95 para que sejam entendidos como indicadores de ajuste suficiente, ao passo que, em relação aos valores do RMSEA, são esperados valores inferiores a 0,08 a fim de que o modelo seja considerado adequado.

Quanto à análise dos coeficientes de Alfa de Cronbach, seguiu-se a orientação de Kline (2000), de modo que valores compreendidos entre 0,60 e 0,70 foram considerados aceitáveis, valores entre $0,70 \mathrm{e}$ 0,90 foram entendidos como bons e valores acima de 0,90 foram interpretados como excelentes.

\section{Resultados}

Considerando o nível de mensuração ordinal, optou-se por utilizar o método denominado Robust Weighted Least Square (WLSMV) (Lara \& Alexis, 2014), de forma que foram testados diferentes modelos, considerando os resultados referentes ao teste de diferença do $\chi^{2}$ entre os modelos unifatorial, oblíquo, de segunda ordem e Bi-factor, para que fosse possível identificar o modelo que apresentasse melhor adequação. Também foram verificadas as diferenças entre os índices de ajustamento: $\chi^{2}$, gl, CFI, TLI e RMSEA. Os resultados podem ser observados na Tabela 2.

Conforme observa-se, os índices obtidos por meio das análises mostram-se muito semelhantes. Entretanto, há discreta superioridade do modelo Bi-factor sobre os demais, uma vez que, nesse modelo, há índices de ajustes mais adequados relacionados ao CFI $(0,951)$ e TLI $(0,942)$, que são interpretados como índices de bom ajuste e ajuste suficiente, respectivamente (Pilati \& Laros, 2007). Por sua vez, o RMSEA $(0,036)$ apresentou valor inferior a 0,08 , de modo que é possível verificar a melhor adequação deste modelo. Outro dado que complementa essa compreensão refere-se à significância dos valores do $\Delta \chi^{2} \mathrm{e}$ $\Delta \mathrm{gl}$, que apontam para o modelo Bi-factor como mais ajustado aos dados, tendo sido selecionado.

Tabela 2

Índices de ajuste confirmatório do modelo unifatorial, oblíquo, segunda ordem e Bi-factor.

\begin{tabular}{lccccccccc}
\hline Modelo avaliado & $\chi^{2}$ & $\mathrm{D} f$ & $\chi^{2} / \mathrm{gl}$ & $\Delta \chi^{2}$ & $\Delta \mathrm{gl}$ & $\mathrm{CFI}$ & $\mathrm{TLI}$ & RMSEA & RMSEA CI 90\% \\
\hline Unifatorial & $537,681^{*}$ & 324 & 1,66 & - & - & 0,942 & 0,937 & 0,038 & $0,032-0,043$ \\
Oblíquo & $497,466^{*}$ & 309 & 1,60 & $40,215^{*}$ & 15 & 0,948 & 0,941 & 0,036 & $0,030-0,042$ \\
Segunda ordem & $524,680^{*}$ & 318 & 1,64 & $27,214^{*}$ & 9 & 0,943 & 0,938 & 0,038 & $0,032-0,043$ \\
Bi-factor & $478,420^{*}$ & 298 & 1,60 & $46,260^{*}$ & 20 & 0,951 & 0,942 & 0,036 & $0,030-0,042$ \\
\hline${ }^{*} \mathrm{p} \leq 0,001$ & & & & & & & & &
\end{tabular}


Após essa constatação, procedeu-se com a análise das cargas fatoriais dos itens para seus fatores teóricos e para o fator geral, conforme apresentado na Tabela 3, a fim de identificar a existência de itens cujas cargas apresentassem valores inferiores a 0,30 , bem como o agrupamento encontrado pelo modelo.

Tabela 3

Valores das cargas fatoriais para os fatores específicos e geral.

\begin{tabular}{|c|c|c|c|c|c|c|c|}
\hline Item & Fator 1 & Fator 2 & Fator 3 & Fator 4 & Fator 5 & Fator 6 & Fator Geral \\
\hline 01 & $-0.090^{* *}$ & & & & & & $0.170^{* *}$ \\
\hline 08 & 0.306 & & & & & & $0.422^{* *}$ \\
\hline 12 & $0.053^{*}$ & & & & & & $0.419^{* *}$ \\
\hline 20 & 0.122 & & & & & & $0.598^{* *}$ \\
\hline 26 & 0.155 & & & & & & $0.571^{* *}$ \\
\hline 02 & & -0.185 & & & & & $0.203^{* *}$ \\
\hline 10 & & 0.080 & & & & & $0.725^{* *}$ \\
\hline 14 & & $0.377^{*}$ & & & & & $0.518^{* *}$ \\
\hline 17 & & $0.377^{*}$ & & & & & $0.808^{* *}$ \\
\hline 03 & & & $0.248^{*}$ & & & & $0.202^{* *}$ \\
\hline 06 & & & 0.177 & & & & $0.531^{* *}$ \\
\hline 13 & & & $0.437^{*}$ & & & & $0.455^{* *}$ \\
\hline 23 & & & -0.197 & & & & $0.669^{* *}$ \\
\hline 27 & & & $0.103^{*}$ & & & & $0.678^{* *}$ \\
\hline 04 & & & & 0.154 & & & $0.557^{* *}$ \\
\hline 07 & & & & 0.227 & & & $0.425^{* *}$ \\
\hline 19 & & & & 0.105 & & & $0.185^{* *}$ \\
\hline 22 & & & & 0.403 & & & $0.259^{* *}$ \\
\hline 11 & & & & & $0.577^{* *}$ & & $0.727^{* *}$ \\
\hline 15 & & & & & $0.252^{* *}$ & & $0.808^{* *}$ \\
\hline 16 & & & & & 0.121 & & $0.590^{* *}$ \\
\hline 24 & & & & & $0.182^{*}$ & & $0.726^{* *}$ \\
\hline 25 & & & & & -0.093 & & $0.594^{* *}$ \\
\hline 05 & & & & & & 0.512 & $0.288^{* *}$ \\
\hline 09 & & & & & & 0.424 & $0.447^{* *}$ \\
\hline 18 & & & & & & -0.109 & $0.276^{* *}$ \\
\hline 21 & & & & & & 0.026 & $0.694^{* *}$ \\
\hline
\end{tabular}

${ }^{*} \mathrm{p} \leq 0,05 ;{ }^{* *} \mathrm{p} \leq 0,01$. Fator $1=$ Vulnerabilidade; Fator 2=Coping; Fator 3=Inteligência Emocional, Fator 4=Bem-estar subjetivo; Fator 5=Locus de controle; Fator 6=Habilidade.

Os resultados da AFC indicaram que, para o instrumento MRI, os itens apresentam duas fontes distintas de variância, ou seja, observa-se que há uma variância específica - relacionada aos seis elementos fundamentais, que a partir deste momento serão chamados fatores, e a uma variância comum que é compartilhada por todos os itens. Ou seja, são observadas cargas fatoriais tanto para um fator geral quanto para os fatores específicos. Assim, o que se pode notar é que os valores das cargas fatoriais dos itens que compõem o Fator 1 - Vulnerabilidade (itens $1,8,12,20$ e 26) variaram de $-0,090$ a 0,306 . Ainda que as cargas destes itens tenham se apresentado baixas para o fator específico, quando analisadas as cargas destes mesmos itens no fator geral, observa-se uma variação de 0,170 a 0,598. Desse 
modo, apenas o item 1 apresentou carga inferior a 0,30 tanto para o fator específico $(-0,090)$ quanto para o fator geral $(0,170)$, motivo pelo qual foi excluído. No que diz respeito ao Fator 2 - Coping (itens 2, 10, 14 e 17), as cargas fatoriais apresentaram variação entre 0,080 e 0,377 e, quando tomados os valores para o fator geral, observou-se variação de 0,203 a 0,808. Para o Fator 2, apenas o item número 2 apresentou valor inferior a 0,30 para o fator específico $(-0,185)$ e para o fator geral $(0,203)$, também excluído do modelo final.

Quando tomados os valores das cargas fatoriais dos itens que compõem o Fator 3 - Inteligência Emocional (itens 3, 6, 13, 23 e 27), pode-se perceber que, para o fator específico, houve variação de 0,103 a $0,437 \mathrm{e}$, para o fator geral, valores que variaram entre 0,202 e 0,678 . Neste conjunto de itens, o de número 3 apresentou valores de 0,248 para o fator específico e de 0,202 para o fator geral, ambos inferiores a 0,30, sendo retirado. Por sua vez, os itens pertencentes ao Fator 4 - Bem-estar subjetivo (4, 7, 19 e 22) apresentaram cargas para o fator específico que variaram entre 0,105 e 0,403 . Quando observados os valores para o fator geral, notou-se variação entre 0,185 e 0,557. Importa ressaltar que o item de número 19 obteve carga de 0,105 para o fator específico e de 0,185 para o fator geral, ambas inferiores a 0,30 , de modo a justificar sua exclusão.
Os itens que compunham o Fator 5 - Locus de controle (item 11, 15, 16, 24 e 25) apresentaram cargas fatoriais que variaram entre - 0,093 e 0,577 para o fator específico, e 0,590 e 0,808 para o fator geral. Nesse conjunto de itens, embora as cargas observadas para o fator específico tenham sido baixas, com exceção do item $11(0,577)$, as cargas do fator geral apresentaram-se adequadas, superiores a 0,30 . E, por fim, os itens que pertenciam ao Fator 6 - Habilidade (5, 9, 18 e 21) apresentaram variação de $-0,109$ a 0,512 para as cargas no fator específico e para o fator geral as cargas variaram entre 0,276 e 0,694 . Nesse conjunto de itens, o de número 18 apresentou carga inferior a 0,30 tanto para o fator específico $(-0,109)$ quanto para o fator geral $(0,276)$ e, por esse motivo, foi retirado da versão final do instrumento.

Considerando que os itens de número 1, 2, 3, 18 e 19 apresentaram cargas inferiores a 0,30 para as condições específicas e geral, motivo pelo qual foram excluídos segundo os critérios adotados (Pilati \& Laros, 2007), uma nova avaliação dos estimadores utilizados inicialmente foi conduzida, com o auxílio do pacote estatístico Mplus 7.11 (Muthén \& Muthén, 2012), a fim de comparar a diferença do $\chi^{2}$ entre o modelo Bi-factor encontrado anteriormente, identificado na Tabela 4 como Bi-factorl, e o modelo $B i$-factor após a exclusão dos cinco itens mencionados, identificado na Tabela 4 como Bi-factor2.

Tabela 4

Comparação dos índices iniciais e posteriores à exclusão dos itens 1, 2, 3, 18 e 19.

\begin{tabular}{lccccccccc}
\hline Modelo avaliado & $\chi^{2}$ & $\mathrm{D} f$ & $\chi^{2} / \mathrm{gl}$ & $\Delta \chi^{2}$ & $\Delta \mathrm{gl}$ & CFI & TLI & RMSEA & RMSEA CI 90\% \\
\hline Bi-factor 1 & $478,420^{*}$ & 298 & 1,60 & - & - & 0,951 & 0,942 & 0,036 & $0,030-0,042$ \\
Bi-factor2 & $264,888^{*}$ & 187 & 1,41 & $213,532^{*}$ & 111 & 0,978 & 0,972 & 0,030 & $0,021-0,038$ \\
\hline${ }^{*} \mathrm{p} \leq 0,001$ & & & & & & & & &
\end{tabular}

Conforme é possível observar, a retirada desses cinco itens contribuiu para a obtenção de melhores índices de ajustes, de modo que a comparação mostrou ajustes superiores aos identificados anteriormente. Tais resultados ampararam a exclusão dos itens 1 (Fator 1 - vulnerabilidade), 2 (Fator 2-coping), 3 (Fator 3-Inteligência Emocional), 18 (Fator 6 - habilidade) e 19 (Fator 4 - bem-estar subjetivo). Dessa forma, a versão final do instrumento MRI ficou composta por 22 itens, dos quais quatro são voltados ao Fator 1 - Vulnerabilidade, três ao Fator 2 Coping, quatro ao Fator 3 - Inteligência emocional, três ao Fator 4 - Bem-estar subjetivo, cinco ao Fator 5 Locus de controle e três ao Fator 6 - Habilidade.

Após essas ações, com o intuito de compreender de que maneira os fatores correlacionavam-se, optou-se por realizar a análise dos coeficientes de correlação de Spearman. A escolha pela análise não paramétrica se deu após a realização do teste de normalidade de Kolmogorov-Smirnov, que se mostrou altamente significativo tanto para os fatores quanto para o total do instrumento, indicando que foi rejeitada a hipótese de normalidade da amostra. Considerando que, segundo 
Aguiar (2007), a interpretação dos valores do coeficiente de Spearman devem ser semelhantes à interpretação dos coeficientes de correlação de Pearson, foram utilizados os critérios apresentados por Valentini e Laros (2012). Estes autores afirmam que devem ser compreendidos como indicadores de efeito pequeno valores inferiores a 0,30 , moderado entre 0,30 e 0,50 , forte entre 0,50 e 0,80 , e superiores a 0,80 como efeito muito forte. Os resultados estão disponibilizados na Tabela 5 .

Conforme é possível observar, foram identificadas correlações positivas e altamente significativas $(p \leq 0,01)$ em todas as interações, com valores de efeitos de forte, média e baixa magnitude. Valores de efeito considerado forte, que variam entre 0,565 e 0,782 , foram encontrados para a interação dos fatores Vulnerabilidade e MRI-Total, Coping e Locus de controle, Coping e MRI-Total, Inteligência emocional e MRI-Total, Locus de controle e MRI-Total e Habilidade e MRI-Total.

Também foram identificadas interações de efeito moderado com valores entre 0,312 e 0,497, para as interações dos fatores Vulnerabilidade e Coping, Vulnerabilidade e Inteligência emocional, Vulnerabilidade e Locus de controle, Coping e Inteligência emocional, Coping e Habilidade, Inteligência emocional e Bem-estar subjetivo, Inteligência emocional e Locus de controle, Inteligência emocional e Habilidade, Bem-estar subjetivo e MRI-Total, e Locus de controle e Habilidade. Por fim, foram identificadas interações de magnitude fraca, com valores variando entre 0,208 e 0,265 , quanto às interações entre os fatores a seguir:Vulnerabilidade e Bem-estar subjetivo, Vulnerabilidade e Habilidade, Coping e Bem-estar subjetivo, Bem-estar subjetivo e Locus de controle, e Bem-estar subjetivo e Habilidade.

Tabela 5

Correlação de Spearman entre os fatores do instrumento MRI.

\begin{tabular}{lccccccc}
\hline & Fator 1 & Fator 2 & Fator 3 & Fator 4 & Fator 5 & Fator 6 & Total \\
\hline Fator 1 & 1 & $0,464^{*}$ & $0,382^{*}$ & $0,228^{*}$ & $0,477^{*}$ & $0,239^{*}$ & $0,689^{*}$ \\
Fator 2 & & 1 & $0,442^{*}$ & $0,208^{*}$ & $0,565^{*}$ & $0,362^{*}$ & $0,719^{*}$ \\
Fator 3 & & & 1 & $0,312^{*}$ & $0,497^{*}$ & $0,454^{*}$ & $0,743^{*}$ \\
Fator 4 & & & & 1 & $0,265^{*}$ & $0,242^{*}$ & $0,464^{*}$ \\
Fator 5 & & & & & 1 & $0,383^{*}$ & $0,782^{*}$ \\
Fator 6 & & & & & & 1 & $0,623^{*}$ \\
MRI - Total & & & & & & & 1 \\
\hline
\end{tabular}

${ }^{*} \mathrm{p} \leq 0,001$. Fator $1=$ Vulnerabilidade; Fator 2=Coping, Fator 3=Inteligência Emocional, Fator 4=Bem-estar subjetivo; Fator $5=$ Locus de controle; Fator 6=Habilidade.

Em seguida, foram realizadas as análises dos coeficientes de alfa de Cronbach para os seis fatores específicos do instrumento, assim como para o total dos itens. Dessa forma, observou-se que os valores obtidos para os fatores Locus de controle $(\alpha=0,751)$ e para o Total dos itens $(\alpha=0,866)$ foram considerados bons, bem como o fator Coping apresentou valor de alpha $(\alpha=0,655)$ considerado aceitável. Os demais fatores apresentaram valores abaixo do esperado, sendo classificados como inaceitáveis: Vulnerabilidade $(\alpha=0,488)$, Inteligência Emocional $(\alpha=0,538)$, Bemestar subjetivo $(\alpha=0,381)$ e Habilidade $(\alpha=0,441)$.

\section{Discussão}

Diante dos desafios da sociedade atual (Faiad et al., 2016), recursos e habilidades associadas ao potencial resiliente mostram-se como competências valiosas, não apenas para o enfrentamento, como também para a apresentação de bons desfechos e condutas adaptativas frente a situações adversas (Masten, 2001, 2018, 2021). Embora exista na literatura o interesse científico na resiliência, ainda não existem instrumentos brasileiros disponíveis para a identificação de seu potencial, especialmente em crianças (Oliveira \& Nakano, 2018, 2019, 2020; Reppold et al., 2012).

Frente a esse cenário, o presente estudo teve dois objetivos: investigar as evidências de validade baseadas na estrutura interna e realizar a investigação da precisão do instrumento MRI (Oliveira \& Nakano, 2020; Oliveira et al., 2019). As ações desenvolvidas seguiram as orientações encontradas na literatura referentes ao caráter contínuo de estudos que visem a investigação 
das qualidades psicométricas dos instrumentos (Ambiel \& Carvalho, 2017; Primi, 2011). Tendo em vista que o MRI foi desenvolvido a partir de um modelo teórico preestabelecido (Castillo et al., 2016) no qual a resiliência é compreendida como o resultado da relação intrínseca entre seis elementos fundamentais, optou-se por conduzir a investigação das evidências de validade baseadas na estrutura interna por meio da Análise Fatorial Confirmatória (AFC), pois tal técnica favorece a compreensão da adequação dos dados empíricos em função da teoria previamente selecionada (Lopes, 2005; Pasquali, 2011).

Portanto, empregou-se a AFC com a finalidade de avaliar, em primeira instância, se o modelo unifatorial seria o mais adequado, pois, conforme apresentado por diversos pesquisadores, como Prince-Embury, Saklofkes e Keefer (2017), os primeiros modelos teóricos acerca do construto defendiam que a resiliência seria um atributo unidimensional. Também considerando que o processo de refinamento dos modelos teóricos voltados à resiliência propuseram, ao longo dos anos, modelos multidimensionais (Castillo et al., 2016; Deep \& Pereira, 2012; Infante, 2007; Junqueira \& Deslandes, 2003; Masten, 2001, 2014, 2018, 2021; Prince-Embury, 2010; Yunes, 2011), buscou-se, também, comparar a adequação dos seguintes modelos multifatoriais: oblíquo, de segunda ordem e Bi-factor, que se diferenciam em função da forma como as variáveis se relacionam entre si e hipoteticamente são alinhados às propostas de modelos teóricos multidimensionais, destacando-se o de Castillo et al. (2016), que sugere que a resiliência é o produto da relação intrínseca entre seis elementos fundamentais.

Os resultados obtidos apresentaram valores muito semelhantes aos índices de ajustes utilizados para a comparação (Lara \& Alexis, 2014; Muthén \& Muthén, 2012; Pilati \& Laros, 2007), entretanto, ligeira superioridade do modelo Bi-factor foi identificada, de modo a sugerir que a estrutura interna do instrumento se desse por meio de seis fatores específicos e de um fator geral. A partir disso, é importante retomar que o modelo teórico de Castillo et al. (2016) sugere que a resiliência - que seria representada empiricamente pelo fator geral - deve ser entendida como o resultado da íntima interação entre os seis elementos fundamentais. Tal pressuposto foi identificado pelo modelo Bi-factor, de modo que o achado empírico se justifica diante do modelo teórico selecionado para o desenvolvimento do instrumento MRI. Na análise realizada, os seis fatores representaram os mesmos elementos fundamentais contidos no modelo original (Castillo et al., 2016), a saber:

- Fator 1-Vulnerabilidade: aspectos pessoais e sociais que possam potencializar a consequência negativa advinda da exposição a uma situação de risco. Invulnerabilidade seria compreendida como $\mathrm{o}$ potencial de diminuir tais consequências negativas.

- Fator 2 - Coping. habilidade cognitiva que favorece o reconhecimento de riscos e o manejo dos problemas a fim de alcançar o alívio ou ainda a solução do problema experimentado. $\mathrm{O}$ enfrentamento pode assumir características positivas que favoreçam a resposta resiliente, ou assumir características negativas que denotarão baixo potencial resiliente de um indivíduo.

- Fator 3 - Inteligência Emocional: considerada como a capacidade dos indivíduos de observar, compreender e regular suas emoções. Reconhece a importância das emoções positivas para o processo de resposta adaptativa.

- Fator 4 - Bem-estar subjetivo: relacionado à noção de felicidade, pois trata-se da capacidade de um indivíduo avaliar suas experiências e afirmar o quanto encontra-se satisfeito. Evidências de relação entre bem-estar subjetivo e altos níveis de resiliência.

- Fator 5 - Locus de Controle: pode ser de caráter interno (quando o indivíduo é capaz de avaliar as contingências de seus comportamentos e atuar frente a situações com intencionalidade) ou externo (quando a avaliação é realizada como consequência externa a seus comportamentos). Quanto mais o sujeito é capaz de apresentar um locus de controle interno, mais resilientes serão suas respostas frente às adversidades.

- Fator6-Habilidade:compreendidacomoautilização de recursos cognitivos para o enfrentamento de problemas, associando o comprometimento a resultados positivos e de excelência.

Em seguida, ainda com o intuito de compreender aspectos relacionados à estrutura interna do instrumento, procedeu-se com a avaliação da correlação estabelecida entre os fatores específicos e, também, da correlação entre os fatores específicos e o fator geral. Dessa forma, o que se nota é que a maioria dos fatores se apresentaram moderadamente correlacionados entre si e fortemente relacionados ao 
fator geral. Tais resultados corroboram a proposta teórica postulada por Castillo et al. (2016), de que a resiliência seja o fruto da relação entre diferentes dimensões (elementos fundamentais) interrelacionadas, que estariam envolvidas no processo de elaboração de uma resposta resiliente, tal como se observa na literatura especializada (Infante, 2007; Masten, 2001, 2014, 2018; Oliveira \& Nakano, 2018, 2019).

É importante ponderar que dentre todos os fatores específicos, o Fator 4 - Bem-estar subjetivo foi o único que apresentou uma correlação de magnitude moderada com o fator geral. Esse fato pode estar relacionado à natureza conceitual desse fator, pois, segundo Castillo et al. (2016), o bem-estar subjetivo pode ser definido como a avaliação positiva que um indivíduo faz sobre sua história de vida, ou seja, trata-se de uma representação de uma avaliação realizada sobre uma variável interna relacionada à satisfação pessoal. Diferentemente das evidências de relação entre bem-estar subjetivo e altos níveis de resiliência que os autores corroboram, tal relação se mostrou a mais fraca no instrumento apresentado. Essa situação merece ser melhor investigada em estudos futuros, de modo a verificar se a magnitude foi influenciada pelos conteúdos dos itens ou se estamos diante de uma especificidade da amostra estudada. Frente aos resultados apresentados, é possível afirmar que as análises realizadas contribuíram não apenas para a compreensão da estrutura do instrumento, mas também permitiram a identificação de itens que deveriam ser excluídos, uma vez que pouco contribuíam para a mensuração da resiliência (Pasquali, 2011; Primi, 2011). Ao final desta etapa, foi possível apresentar a versão final do MRI, composto, então, por 22 itens.

Ainda no que se refere aos objetivos deste estudo, é importante considerar que, além da busca pelas evidências de validade do instrumento, é importante verificar se a avaliação realizada é livre o suficiente do chamado "erro de medida", usualmente estimado por meio da precisão (Ambiel \& Carvalho, 2017). Segundo os autores, a avaliação de construtos psicológicos envolve erro de medida, sendo, no entanto, esperado que essa quantidade seja limitada e conhecida. Isso porque quanto maior o erro de medida, menor a precisão de instrumento.

Neste estudo, optou-se pela utilização do coeficiente Alfa de Cronbach, possivelmente o método mais utilizado e usualmente empregado em testes baseados em escala Likert e de avaliação da personalidade e atitude (Zanon \& Hauck Filho, 2015). Os resultados apontaram para a adequação dos fatores específicos Fator 2 - Coping, Fator 5 - Locus de controle e do fator geral. Eles apresentaram coeficientes considerados bons e aceitáveis. Os demais fatores específicos apresentaram valores considerados não aceitáveis.

Entretanto, é importante considerar que, segundo Namdeo e Rout (2016) e Tavakol e Dennick (2011), há limitações relacionadas aos estudos que envolvem a análise dos coeficientes de alfa, quando esta estratégia é utilizada para aferir a precisão de instrumentos curtos. Segundo esses autores, instrumentos com poucos itens podem apresentar valores de alfa inferiores ainda que apresentem outros tipos de evidências de precisão - como a estabilidade temporal - os quais devem ser investigados posteriormente. Considerando a faixa etária almejada neste instrumento, optou-se por um número reduzido de itens, a fim de que a tarefa não se tornasse demasiadamente cansativa para os participantes, portanto, é possível que essa escolha tenha influenciado os resultados obtidos nesta análise, em especial quanto aos fatores 1 - Vulnerabilidade, 3 - Inteligência emocional, 4 - Bem-estar subjetivo e 6 - Habilidade.

\section{Considerações finais}

Estudos que busquem investigar as qualidades psicométricas de instrumentos em processo de construção são importantes para que exista maior compreensão dos potenciais e limitações dos materiais. O presente estudo, considerando lacunas identificadas na literatura, se propôs a investigar as evidências de validade baseadas na estrutura interna e precisão de um instrumento para avaliação da resiliência na infância. Em conjunto, as análises realizadas permitiram uma visão mais ampla de como os itens do instrumento Marcadores de Resiliência Infantil se organizam, bem como confirmaram o modelo teórico adotado, apontando resultados favoráveis a algumas qualidades psicométricas do instrumento em processo de construção. Diante desses resultados, é possível afirmar que foram alcançadas evidências iniciais relacionadas à estrutura interna e à precisão do instrumento. Embora sejam conclusões favoráveis, é oportuno refletir que limitações se fizeram presentes ao longo deste estudo. Dentre elas, destaca-se a ausência de variabilidade da amostra quanto aos estados e regiões do país, uma vez que todos os participantes eram provenientes da 
região Sudeste, mais especificamente, do interior do estado de São Paulo. Dessa forma, cabe considerar a necessidade de que mais estudos sejam conduzidos com o instrumental, até que se atinja segurança acerca dos seus resultados. Outrossim, é importante que outras investigações busquem averiguar outros aspectos junto ao instrumento. Assim, sugere-se que futuramente sejam conduzidos estudos que avaliem os itens do MRI por meio de Teoria de Resposta ao Item e que investiguem outras fontes de precisão, tais como estabilidade temporal, utilizando o delineamento de teste e reteste.

\section{Referências}

Aguiar, P. (2007). Estatística em investigação epidemiológica: SPSS: Guia prático de medicina. Climepsi Editores.

Ambiel, R. A. M., \& Carvalho, L. F. (2017). Validade e precisão de instrumentos de avaliação psicológica. In M. R. C. Lins \& J. C. Borsa (Orgs.), Avaliação psicológica: Aspectos teóricos e práticos (pp. 115-125). Vozes.

Castillo, J. A. G., Castillo-López, A. G., López-Sánchez, C., \& Dias, P. C. (2016). Conceptualización teórica de la resiliencia psicosocial y su relación con la salud. Health and Addictions, 16(1), 59-68.

Deep, C. A. F. N., \& Pereira, I. (2012). Adaptação da "The Resilience Scale" para a população adulta portuguesa. Psicologia USP, 23(2), 417-433. https://doi.org/10.1590/S0103-65642012005000008

Faiad, C., Pasquali, L., \& Primi, R. (2016). Construção e evidências de validade do Teste de Reação à Frustração Objetivo. Psicologia: Teoria e Pesquisa, 32(spe). https://doi.org/10.1590/0102-3772e32ne224

Fontes, A. P. (2010). Resiliência, segundo o paradigma do desenvolvimento ao longo da vida (life-span). Kairós, 7(1), 8-20. https://revistas.pucsp.br/index.php/kairos/article/view/3917

Infante, F. (2007). A resiliência como processo: uma revisão da literatura recente. In A. Melillo \& E. N. S. Ojeda (Orgs.), Resiliência: descobrindo as próprias fortalezas (pp. 23-38). Artmed.

Junqueira, M. F. P. S., \& Deslandes, S. F. (2003). Resiliência e maus tratos à criança. Cadernos de Saúde Pública, 19(1), 227-235. https://doi.org/10.1590/S0102-311X2003000100025

Kline, P. (2000). The handbook of psychometric testing (2a ed.). Routledge.

Lara, D., \& Alexis, S. (2014). ¿Matrices Policóricas/Tetracóricas o Matrices Pearson? Un estudio metodológico. Revista Argentina de Ciencias del Comportamiento, 6(1), 39-48.

Lopes, H. E. G. (2005). Abrindo a caixa preta: considerações sobre a utilização da análise fatorial confirmatória nas pesquisas em administração. E \& G: Economia e Gestão, 5(11), 19-34. http://periodicos.pucminas.br/index.php/ economiaegestao/article/view/42

Luthar, S., Cicchetti, D., \& Becker, B. (2000). The construct of resilience: a critical evaluation and guidelines for future work. Child Development, 71(3), 543-558. https://www.ncbi.nlm.nih.gov/pmc/articles/PMC1885202/

Masten, A. S. (2001). Ordinary magic: resilience processes in development. American Psychologist, 56(3), 227-238. https://doi.org/10.1037/0003-066X.56.3.227

Masten, A. S. (2014). Global perspectives on resilience in children and youth. Child Development, 85(1), 6-20. https://doi.org/10.1111/cdev.12205

Masten, A. S. (2018). Resiliency theory and research on children and families: past, present, and promise. Journal of Family Theory \& Review, 10(1), 12-31. https://doi.org/10.1111/jftr.12255

Masten, A. S. (2021). Resilience of children in disasters: a multisystem perspective. International Journal of Psychology, 56(1), 1-11. https:// doi.org/10.1002/ijop.12737

Masten, A. S., \& Barnes, A. J. (2018). Resilience in children: developmental perspectives. Children, 5(7), 98. https://doi.org/10.3390/children5070098

Masten, A. S., \& Cicchetti, D. (2016). Resilience in development: progress and transformation. In D. Cicchetti (Ed.), Developmental psychopathology: risk, resilience, and intervention (3a ed., Vol. 4, pp. 271-333). John Wiley \& Sons.

Muthén, L. K., \& Muthén, B. O. (2012). Mplus: Statistical Analysis With Latent Variables: User's Guide (7a ed.). Muthén \& Muthén. 
Namdeo, S. K., \& Rout, S. D. (2016). Calculating and interpreting Cronbrach's alpha using Rosenberg assessment scale on pediatrician's attitude and perception on self-esteem. International Journal of Community Medicine and Public Health, 3(6), 1371-1374. https://doi.org/10.18203/2394-6040.ijcmph20161448

Oliveira, K. S., \& Nakano, T. C. (2018). Avaliação da resiliência em psicologia: revisão do cenário científico brasileiro. Psicologia em Pesquisa, 12(1), 1-11. https://doi.org/10.5327/Z1982-1247201500020003

Oliveira, K. S., \& Nakano, T. C. (2019). Avaliação da resiliência: uma revisão internacional. Psicologia em Revista, 25(3), 1021-1043. https://doi.org/10.5752/P.1678-9563.2019v25n3p1021-1043

Oliveira, K. S., \& Nakano, T. C. (2020). Desenvolvimento e investigação de evidências de validade para o instrumento Marcadores de Resiliência Infantil. Psico-USF, 25(4), 737-749. https://doi.org/10.1590/1413/82712020250412

Oliveira, K. S., Nakano, T. C., \& Silva, G. M. (2019). Children's Resilience Markers: Criterion-based validity evidences. Estudos de Psicologia (Campinas), 36. https://doi.org/10.1590/1982-0275201936e180142

Oliveira, L. L., Costa,V.M.R., Requeijo, M. R., Rebolledo, R. S., Pimenta, A. F., \& Lemos, S. M. A. (2012). Desenvolvimento infantil: concordância entre a caderneta de saúde da criança e o manual para vigilância do desenvolvimento infantil. Revista Paulista de Pediatria, 30(4), 479-485. https://doi.org/10.1590/S0103-05822012000400004

Pasquali, L. (Ed.). (2011). Técnicas do exame psicológico - TEP: manual. Casa do Psicólogo; Conselho Federal de Psicologia.

Pilati, R., \& Laros, J. A. (2007). Modelos de equações estruturais em psicologia: conceitos e aplicações. Psicologia: Teoria e Pesquisa, 23(2), 205-216. https://doi.org/10.1590/S0102-37722007000200011

Poletto, M., Wagner, T. M. C., \& Koller, S. H. (2004). Resiliência e desenvolvimento infantil de crianças que cuidam de crianças: uma visão em perspectiva. Psicologia: Teoria e Pesquisa, 20(3), 241-250. https://doi.org/10.1590/ S0102-37722004000300005

Primi, R. (2011). Responsabilidade ética no uso de padrões de qualidade profissional na avaliação psicológica. In Conselho Federal de Psicologia (Org.), Ano da Avaliação Psicológica - Textos Geradores (pp. 53-58). Conselho Federal de Psicologia.

Prince-Embury, S. (2006). Resiliency Scales for Children and Adolescents: A profile of personal strengths. Harcourt Assessment.

Prince-Embury, S. (2010). Psychometric properties of the Scales for Resiliency for Children and Adolescents and use for youth with psychiatric disorders. Journal of Psychoeducational Assessment, 28(4), 291-302. https://doi.org/ $10.1177 / 0734282910366832$

Prince-Embury, S., Saklofkes, D. H., \& Keefer, K. V. (2017). Three-factor model of personal resiliency. In U. Kumar (Ed.), The routledge international handbook of psychosocial resilience (pp. 119-140). Routledge.

Reppold, C. T., Mayer, J. C., Almeida, L. S., \& Hutz, C. S. (2012). Avaliação da resiliência: Controvérsia em torno do uso de escalas. Psicologia: Reflexão e Crítica, 25(2), 248-255. https://doi.org/10.1590/S0102-79722012000200006

Tavakol, M., \& Dennick, R. (2011). Making sense of Cronbach's alpha. International Journal of Medical Education, 2, 53-55. https://doi.org/10.5116/ijme.4dfb.8dfd

Valentini, F., \& Laros, J. A. (2012). Métodos atuais de estatística aplicada e psicometria. In C. S. Hutz (Org.), Avanços em avaliação psicológica e neuropsicológica de crianças e adolescentes II (pp. 7-40). Casa do Psicólogo.

Yule, K., Houston, J., \& Grych, J. (2019). Resilience in children exposed to violence: a meta-analysis of protective factors across ecological contexts. Clinical Child Fam Psychology Review, 22, 406-431. https://doi.org/10.1007/ s10567-019-00293-1

Yunes, M. A. M. (2011). Psicologia positiva e resiliência: foco no indivíduo e na família. In D. D. Dell'Aglio, S. H. Koller \& M. A. M. Yunes (Orgs.), Resiliência e psicologia positiva: Interfaces do risco à proteção (pp. 45-68). Casa do Psicólogo.

Zanon, C., \& Hauck Filho, N. (2015). Fidedignidade. In C. S. Hutz, D. H. Bandeira \& C. M. Trentini (Orgs.), Psicometria (pp. 85-95). Artmed. 


\section{Karina da Silva Oliveira}

Docente do curso de pós-graduação stricto sensu em Psicologia: Cognição e Comportamento, e do departamento de psicologia da Universidade Federal de Minas Gerais, Belo Horizonte - MG. Brasil. Pesquisadora da linha de mensuração e intervenção em psicologia. Pós-doutorado na Universidade São Francisco, Campinas - SP. Brasil. Psicóloga, mestre e doutora em Psicologia, pela Pontifícia Universidade Católica de Campinas (PUC-Campinas), Campinas-SP. Brasil. Especialista em Neuropsicologia Aplicada à Neurologia Infantil pela Universidade Estadual de Campinas (UNICAMP), Campinas - SP. Brasil. Atua nas áreas de avaliação psicológica, construção de instrumentos, infância, criatividade e resiliência. Membro do Laboratório de Avaliação e Intervenção na Saúde (LAVIS), membro do grupo de trabalho Avaliação Psicológica em Psicologia Positiva e Criatividade na Anpepp e membro da diretoria do Instituto Brasileiro de Avaliação Psicológica (2021-2023).

E-mail: karina_oliv@yahoo.com.br

(1) https://orcid.org/0000-0002-5301-7012

\section{Tatiana de Cássia Nakano}

Docente do curso de pós-graduação stricto sensu em Psicologia da Pontifícia Universidade Católica de Campinas (PUC-Campinas), Campinas-SP. Brasil. Pesquisadora da linha de Instrumentos e processos em avaliação psicológica. Pós-doutorado na Universidade São Francisco (USF), Bragança Paulista - SP. Brasil. Doutorado em Psicologia como Profissão e Ciência pela Pontifícia Universidade Católica de Campinas (PUC-Campinas), Campinas - SP. Brasil. Atua principalmente nas áreas de Avaliação Psicológica, Criatividade, Altas Habilidades/Superdotação, Inteligência, Competências Socioemocionais, Psicologia do Esporte e Psicologia Positiva. Presidente passado da Associação Brasileira de Criatividade e Inovação (Criabrasilis, 2014-2017), membro colaborador do Conselho Brasileiro para Superdotação (Conbrasd, 2018-2020) e membro do grupo de trabalho Psicologia Positiva e Criatividade na Anpepp. E-mail: tatiananakano@hotmail.com

(D) https://orcid.org/0000-0002-5720-8940

\section{Evandro Morais Peixoto}

Docente do Programa de Pós-Graduação Stricto Sensu em Psicologia da Universidade São Francisco (USF), Campinas - SP. Brasil. Bolsista de Produtividade CNPQ PQ2. Psicólogo pela Universidade Presbiteriana Mackenzie. Mestre e Doutor em Psicologia como Profissão e Ciência pela Pontifícia Universidade Católica de Campinas (PUCCampinas), Campinas - SP. Brasil. Estágio doutoral desenvolvido na Université du Québec à Trois-Rivières (UQTR), QC. Canadá. Estágio pós-doutoral desenvolvido na USF. Professor visitante na Université du Québec à Montreal (UQAM). Canada. Membro do GT Avaliação Psicológica em Psicologia Positiva e Criatividade na Anpepp. Membro da Comissão Consultiva em Avaliação Psicológica CCAP/CFP (2020-2022). Principais temas de investigação: Psicologia do esporte, Avaliação Psicológica, Psicometria, Desenvolvimento Positivo de Jovens no Esporte. Coordenador do Núcleo de Estudo e Pesquisa em Psicologia do Esporte e do Exercício (NuEPPEE).

E-mail: epeixoto_6@hotmail.com

(1) https://orcid.org/0000-0003-1007-3433

Endereço para envio de correspondência:

Laboratório de Avaliação e Intervenção na Saúde (LAVIS-UFMG). Av. Presidente Antonio Carlos, 6627, sala 4006, Bairro Campus Pampulha. CEP: 31270-901. Belo Horizonte - MG. Brasil.

Recebido 07/03/2019

Aceito 12/03/2021

Received 03/07/2019

Approved 03/12/2021

Recibido 07/03/2019

Aceptado 12/03/2021 
Como citar: Oliveira, K. S., Nakano, T. C., \& Peixoto, E. M. (2021). Marcadores de Resiliência Infantil: Evidências de Validade para Estrutura Interna e Precisão. Psicologia: Ciência e Profissão, 41, 1-15. https://doi.org/10.1590/ 1982-3703003220979

How to cite: Oliveira, K. S., Nakano, T. C., \& Peixoto, E. M. (2021). Children's Resilience Markers: Validity Evidence for Internal Structure and Reliability. Psicologia: Ciência e Profissão, 41, 1-15. https://doi.org/10.1590/ $1982-3703003220979$

Cómo citar: Oliveira, K. S., Nakano, T. C., \& Peixoto, E. M. (2021). Marcadores de Resiliencia Infantil: Evidencias de Validez para la Estructura Interna y Exactitud. Psicologia: Ciência e Profissão, 41, 1-15. https://doi.org/10.1590/ 1982-3703003220979 